\title{
Representation of motor habit in a sequence of repetitive reach and grasp movements performed by macaque monkeys: Evidence for a contribution of the dorsolateral prefrontal cortex
}

\author{
Mélanie Kaeser ${ }^{a, b}$, Thierry Wannier ${ }^{a}$, Jean-François Brunet ${ }^{b}$, Alexander Wyss ${ }^{a}$, \\ Jocelyne Bloch ${ }^{b}$ and Eric M. Rouiller ${ }^{a, *}$ \\ ${ }^{a}$ Department of Medicine and Fribourg Center for Cognition, University of Fribourg, Fribourg, Switzerland \\ ${ }^{\mathrm{b}}$ Department of Neurosurgery, Lausanne University Hospital (CHUV), Lausanne, Switzerland
}

\begin{abstract}
In the context of an autologous cell transplantation study, a unilateral biopsy of cortical tissue was surgically performed from the right dorsolateral prefrontal cortex (dlPFC) in two intact adult macaque monkeys (dlPFC lesioned group), together with the implantation of a chronic chamber providing access to the left motor cortex. Three other monkeys were subjected to the same chronic chamber implantation, but without dlPFC biopsy (control group). All monkeys were initially trained to perform sequential manual dexterity tasks, requiring precision grip. The motor performance and the prehension's sequence (temporal order to grasp pellets from different spatial locations) were analysed for each hand. Following the surgery, transient and moderate deficits of manual dexterity per se occurred in both groups, indicating that they were not due to the dlPFC lesion (most likely related to the recording chamber implantation and/or general anaesthesia/medication). In contrast, changes of motor habit were observed for the sequential order of grasping in the two monkeys with dlPFC lesion only. The changes were more prominent in the monkey subjected to the largest lesion, supporting the notion of a specific effect of the dlPFC lesion on the motor habit of the monkeys. These observations are reminiscent of previous studies using conditional tasks with delay that have proposed a specialization of the dlPFC for visuo-spatial working memory, except that this is in a different context of "free-will", nonconditional manual dexterity task, without a component of working memory.
\end{abstract}

\section{Introduction}

A human subject faces for instance the behavioural task to collect strawberries from plants arranged along rows and columns in a garden zone of a few square meters. Although one can pick-up the fruits in a random order without following a systematic spatio-temporal sequence, most people would choose to follow a well-defined sequence (e.g., from top row to

\footnotetext{
* Corresponding author.

E-mail address: eric.rouiller@unifr.ch (E.M. Rouiller).
} 
bottom row and/or from left column to right column or viceversa). When first confronted to the task, a systematic order in the picking sequence derives from a motor strategy aimed at optimizing the task (e.g., to not miss a few plants, a risk inherent to a random exploration). When the same task is repeated over and over, then the motor strategy turns into a motor habit, in which the subject does not re-think to optimize the task. In this context, it is legitimate to address where such motor habit related to over-trained and nearly automatic sequential motor task is represented in the brain.

There are numerous reports from non-human primate studies emphasizing that motor sequencing of voluntary ocular or arm movements is represented in the mesial part of the motor cortex (in the large sense), in particular in the supplementary motor area (SMA), in both the caudal SMAproper and the rostal pre-SMA (e.g., Mushiake et al., 1990, 1991; Tanji and Shima, 1994; Clower and Alexander, 1998; Shima and Tanji, 1998, 2000; Isoda and Tanji, 2003, 2004; Sohn and Lee, 2007; Berdyyeva and Olson, 2010). In the behavioural paradigms applied in these studies, the temporal sequence of movements was instructed visually and, in most cases, memorized by the monkeys so that it could be repetitively reproduced, before moving on to another sequence of targets, and so on. Still in monkeys (Barone and Joseph, 1989; Funahashi et al., 1993; Ninokura et al., 2004; Shima et al., 2007; Berdyyeva and Olson, 2010), a fairly comparable representation of motor sequencing was found in the dorsolateral prefrontal cortex (dlPFC). A role played by SMA in the control of motor sequences has also been found in human subjects (e.g., Gerloff et al., 1997; Boecker et al., 1998; Deiber et al., 1999; Lepage et al., 1999; Schubotz and Von Cramon, 2001; Verwey et al., 2002; Van Mier et al., 2004; Bengtsson et al., 2004; Kennerley et al., 2004; Tanaka et al., 2010).

In the monkey and human experiments listed above, the motor sequence was strictly controlled (visual guidance and/ or memorized) and, in most cases, highly practiced if not over-trained. Although the sequential task of strawberry picking introduced as example in the first paragraph above may also be over-practiced, it is fundamentally different in the sense that it is a "free-will" motor performance without imposed sequential order. Furthermore, there is neither visual guidance nor memorization (at least in the working memory) of the motor sequence. The neural representation of such motor habit underlying a motor sequence performed mainly automatically is poorly understood. To address this issue, adult monkeys were trained to perform repetitive manual dexterity tasks (derived from previous versions: see Brinkman and Kuypers, 1973; Brinkman, 1984), comprising a spatial component and a temporal sequence, thus possibly implicating dlPFC, at least during the training phase (Shima et al., 2007). Nevertheless, contrarily to most, if not all, studies on dlPFC (e.g., Barone and Joseph, 1989; Ninokura et al., 2004; Shima et al., 2007), which are built on conditional tasks with delay and emphasize on the retention of a pertinent information (spatial) used to execute the sequential task correctly, the present results are based on "free-will" manual dexterity tasks (see Schmidlin et al., 2011). The manual tasks require a sequence of repetitive movements aimed at different locations, in absence of any constraint of success, temporal order or pace. In other words, the motor tasks used in the present study are nonconditional, without delay, thus not implicating working memory per se once learning or practice is acquired. In this context, after a stable motor performance was reached, thus excluding the learning phase, a unilateral biopsy of cortical tissue was performed surgically in the right dlPFC of intact monkeys, with the aim to culture progenitor cells in order to perform subsequently an autologous reimplantation into the lesioned motor cortex (Kaeser et al., 2011). We hypothesized that in such situation of over-trained sequential movements based on motor habit, the lesion of dlPFC was performed at a site that is non-pertinent for the motor control itself (manual dexterity performance), but it remains unclear whether the dlPFC still plays a role in the representation of motor habits governing the sequential order of repetitive manual dexterity movements to be performed to complete an over-trained motor task executed on a "free-will" basis. Addressing this issue for dlPFC is also prompted by a previous observation also in macaques that a lesion of an adjacent cortical area (SMA) led to a change of motor sequence for a similar manual dexterity task (Brinkman, 1984).

Considering dlPFC as a candidate for such motor habit representation is consistent with the multisensory inputs reaching dlPFC and its projections to brain structures with motor functions, such as the premotor cortex, the superior colliculus, and the basal ganglia. Premotor areas, in turn, project to the primary motor cortex and to the spinal cord (Bates and Goldman-Rakic, 1993; Dum and Strick, 1991, 2005; Fang et al., 2006; Kurata, 1991; Leichnetz, 1986; Lu et al., 1994; Matelli et al., 1986; Rizzolatti and Luppino, 2001; Sakagami and Watanabe, 2007). It has been demonstrated that PFC, in particular dlPFC, plays a crucial role in motor learning and in the intuitive optimization of a task (establishment of a strategy), whereas its implication seems to decrease as the task is progressively trained and automated, delegating the responsibility of the realization of this acquired specific motor task to other brain regions, such as basal ganglia, premotor, primary motor, supplementary motor and cingulate motor areas (see for review Hadj-Bouziane et al., 2003; Halsband and Lange, 2006; Passingham, 1996). Nevertheless, one cannot exclude that dlPFC remains engaged in a neural network underlying motor habits adopted to perform a "free-will" sequential motor task.

\section{Methods}

\subsection{Subjects and behavioural tasks}

For the present study, data were collected from a group of 5 male long-tailed macaques (Macaca fascicularis), weighting between 3 and $6 \mathrm{~kg}$ (Mk-VA, Mk-SL, Mk-JA, Mk-JO, Mk-AV). The monkeys ranged from 2.5 to 5.5 years old at the time of initiation of behavioural training sessions. All the behavioural and surgical procedures were approved by the local ethical committee, in accordance with the Guidelines for the Care and Use of Laboratory Animals and approved by Swiss veterinary authorities (see e.g., Kaeser et al., 2010, 2011; Bashir et al., 2012; Schmidlin et al., 2011). Briefly, the monkeys were trained to enter and sit into a Plexiglas primate chair 
(Schmidlin et al., 2011). On the front panel of the primate chair, two sliding doors allowed testing separately the left hand or the right hand in various manual dexterity tasks (Schmidlin et al., 2011). The monkeys were not food deprived and had free access to water, but they did not eat before the training sessions on that day. After each task, the monkeys received supplemental food as positive reinforcement, such as dried raisins, almonds, hazelnuts, peanuts, and cereals. The body weight of the animals was monitored prior to each daily behavioural session. A criterion of interruption of the experiments was a loss of $10 \%$ of the total weight of the animal, an event that did not occur. For the present investigation, the motor performances at the modified Brinkman board task - static - and the rotating Brinkman board task moving either clockwise or counterclockwise - were analysed. These two tasks were described in more detail in previous studies (Brinkman and Kuypers, 1973; Brinkman, 1984; Liu and Rouiller, 1999; Freund et al., 2009; Kaeser et al., 2010, 2011; Schmidlin et al., 2011) and can be seen on the following web page: http://www.unifr.ch/neuro/rouiller/ research/brink.php.

For each monkey, the performance of the left hand and the right hand were analysed separately. Analyses were conducted on 11-15 recorded sessions before PFC lesion and/or cortical chamber implantation, as well as 11-15 recorded sessions after PFC lesion and/or cortical chamber implantation. The behavioural sessions were analysed with a video recorder allowing frame by frame analysis, with a resolution of 25 frames per second. The following parameters were analysed: (i) The score, given by the number of pellets successfully grasped in $30 \mathrm{sec}$, analysed only for the modified Brinkman board task; (ii) The contact time, which is the time interval between the first digit contact with the pellet in the well and the removal of the successfully grasped pellet from the well. For the modified Brinkman board task, this parameter was analysed for the 5 first and 5 last vertical and horizontal visited wells ( 20 wells in total). For the rotating Brinkman board task, this parameter was analysed on all wells ( $n=32$ ); (iii) The sequence of prehension, defined as the order according to which the animal visited the different wells to grasp the food pellets. For the modified Brinkman board task, each well was numbered (Fig. 1A), whereas for the rotating Brinkman board task, the ring position (4 in total) was taken into account (Fig. 1B). The temporal sequence was then quantified as explained in the results section in order to determine whether the monkeys adopted a reproducible temporal sequence for visiting the different wells.

These data were analysed and coded in an analysis protocol sheet and a database was then created in an Excel spreadsheet. Graphics, panels and statistical analyses were performed using the softwares Excel, and SigmaPlot/SigmaStat. Some graphs were also produced with a home-made program developed in Matlab. The statistical analysis was based on the distribution of motor performance data points post-surgery with respect to a domain of reference defined by mean pre-surgery values plus or minus 2 standard deviations (SDs) (e.g., Fig. 3). The quantitative data reflecting the motor sequences were analysed statistically based on the nonparametric Mann and Whitney test, again comparing preversus post-surgery data (e.g., Figs. 5 and 7).
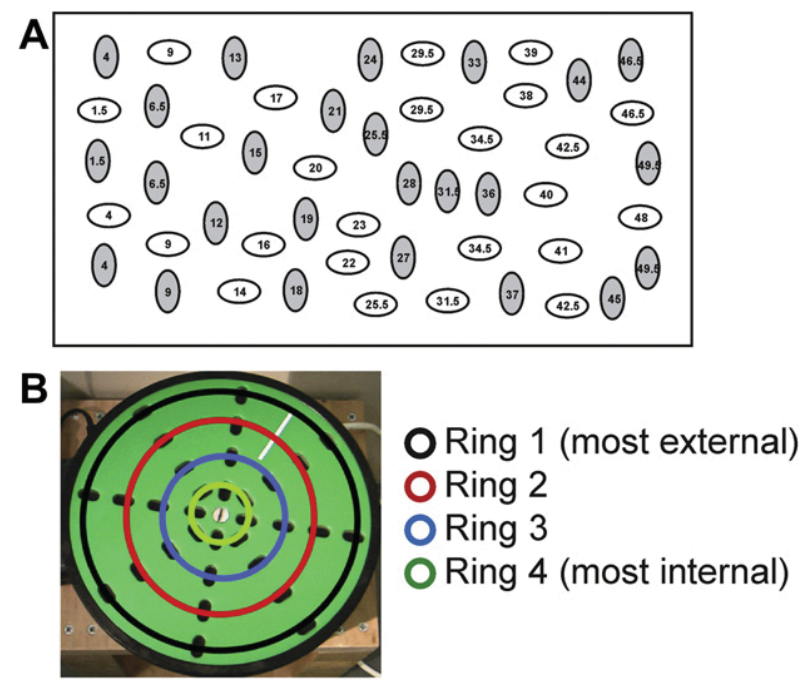

Fig. 1 - The modified Brinkman board - static- (panel A), represented here as seen from above, contains 25 vertical wells and 25 horizontal wells. As the monkeys usually scanned the board following an ordered sequence along the horizontal axis and not along the vertical axis, the wells were numbered according to their position going from left to right (numbers 1.5-49.5). When two wells have a similar left to right coordinate (e.g., the most left 2 wells), their sequential number was set to 1.5 for both. The next three wells (3, 4 and 5) having comparable coordinates, their sequential number was set to 4 for all of them. The rotating Brinkman board -moving either clockwise or counterclockwise- (panel B), also as seen from above, is organized in four concentric rings of wells, ranging from 1 (most external) to 4 (most internal).

\subsection{Surgical procedures}

The surgical procedures were described in previous reports from this laboratory (Liu and Rouiller, 1999; Kaeser et al., 2010, 2011; Bashir et al., 2012). Briefly, after completion of training and once the monkey reached a behavioural plateau, the animal was subjected to the following surgical procedures. The operative site was shaved and cleaned with betadin and then with alcohol. For all surgeries, the monkey was first tranquilized with ketamine (Ketalar ${ }^{\circledR}$; Parke-Davis, $5 \mathrm{mg} / \mathrm{kg}$, intramusculary) and atropine was injected $(.05 \mathrm{mg} / \mathrm{kg}$, intramusculary) in order to reduce bronchial secretions. Before surgery, the animal was treated with the analgesic Carprofen (Rymadil $^{\circledR}, 4 \mathrm{mg} / \mathrm{kg}$, subcutaneously) and the antibiotic Albipen $^{\circledR}$ (Ampiciline 10\%, $30 \mathrm{mg} / \mathrm{kg}$, subcutaneously). Subsequently, the monkey was anaesthetized with intravenous perfusion of $1 \%$ propofol (Fresenius ${ }^{\circledR}$ ) mixed with a $5 \%$ glucose solution (1 volume of propofol and 2 volumes of glucose solution); ketamine was added to the perfusion solution $(65 \mathrm{mg} / 100 \mathrm{ml})$. To prevent brain oedema, Methylprednisolone (Solu-medrol, Pfizer ${ }^{\circledR}$ ) was added to the propofol/ glucose solution $(1 \mathrm{mg} / \mathrm{ml})$. The level of anaesthesia was kept at an optimal level with a perfusion rate of the propofol/ glucose mixture of $0.1 \mathrm{ml} / \mathrm{min} / \mathrm{kg}$. 
Biopsied Mk-JO

A
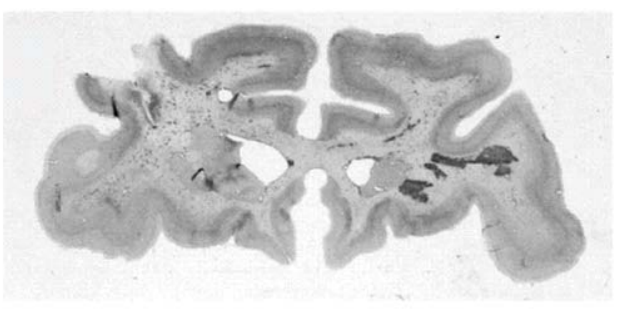

B

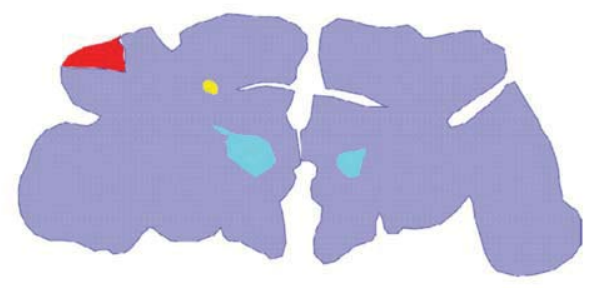

C

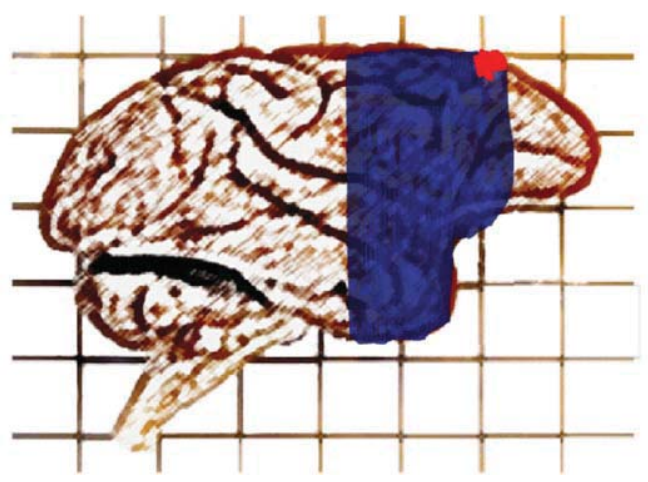

\section{Biopsied Mk-AV}
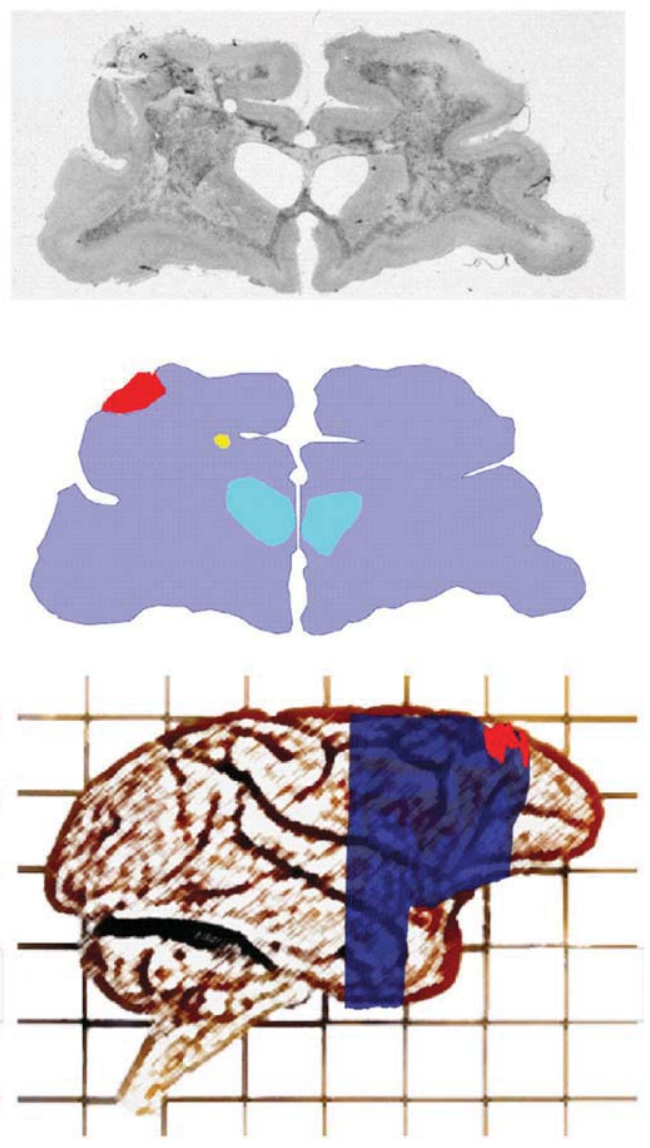

Fig. 2 - A. Nissl-stained sections in the frontal plane of the dlPFC lesioned monkeys' brain, at the level of the centre of the biopsy along the rostro-caudal axis. B. Reconstructions of the biopsy performed from Nissl-stained sections in order to define its size and location. The biopsy is represented in red, the ventricles are in turquoise, whereas the yellow circle corresponds to the pipette passing through the whole brain rostro-caudally and allowing to align the brain sections for 3D reconstruction, represented in C (blue area), superimposed on a standard macaque monkey brain illustration.

In the surgery room, the animal was placed in a stereotaxic framework, with local anaesthetics put on the ear bars in order to reduce pain possibly originating from the ear canals. Before the incision, the operative site was covered with antimicrobial incision drape (Steri-drape $3 \mathrm{M}^{T M} \operatorname{Ioban}^{\mathrm{TM}} 2$ ) to thoroughly dry the intact skin. All surgeries were performed in a facility approved by the local cantonal veterinary officer, with strict attention to sterile techniques. Heart rate, respiration rate, expired $\mathrm{CO}_{2}$, arterial $\mathrm{O}_{2}$ saturation and rectal temperature were carefully monitored throughout the surgery.

All monkeys included in the present study $(n=5)$ were subjected to a craniotomy above the motor cortex in order to implant a chronic rectangular stainless steel recording/stimulating chamber for electrophysiological investigations over the forelimb area of the motor cortex on the left hemisphere, as previously described (e.g., Liu and Rouiller, 1999). The recording chamber was anchored to the skull with titanium screws (Synthes ${ }^{\circledR}$, Cortex screw). The dura mater was kept intact. The whole implant was secured to the skull with dental acrylic cement and/or with orthopaedic cement (Palacos ${ }^{\circledR} 40$ Gentamicin $500 \mathrm{mg}$ ). The size of the chronic recording chamber was $22 \times 17 \times 15 \mathrm{~mm}$ for three monkeys (Mk-JO, Mk$\mathrm{JA}$ and Mk-AV), and $28 \times 19 \times 17 \mathrm{~mm}$ for the other two monkeys (Mk-VA and Mk-SL). At the end, the skin around the chamber was sutured.

On the surgery day when the chamber was implanted, two monkeys only ( $\mathrm{Mk}-\mathrm{JO}$ and $\mathrm{Mk}-\mathrm{AV}^{1}$ ) were also subjected to a right dlPFC biopsy which was performed as follows. A $8 \mathrm{~mm} \times 8 \mathrm{~mm}$ craniotomy was performed above the right dlPFC and the dura mater was incised. Then, a piece of cortical tissue was extracted using a surgical blade (no. 11, Paragon ${ }^{\circledR}$ ). In contrast to the chronic recording chamber above M1, the bone flap above dlPFC was put back in place and sutured to the skull. The muscle and the skin were then sutured as well. As

\footnotetext{
${ }^{1}$ Mk-JA appeared in previous reports (Kaeser et al., 2010, 2011) as a monkey subjected to an autologous transplantation of progenitor cells derived from dlPFC biopsy. However, the biopsy in dlPFC of Mk-JA took place at a later time point with respect to chronic chamber implantation. For this reason, Mk-JA appears here as a control monkey subjected to a chronic chamber implantation only at a given time point.
} 

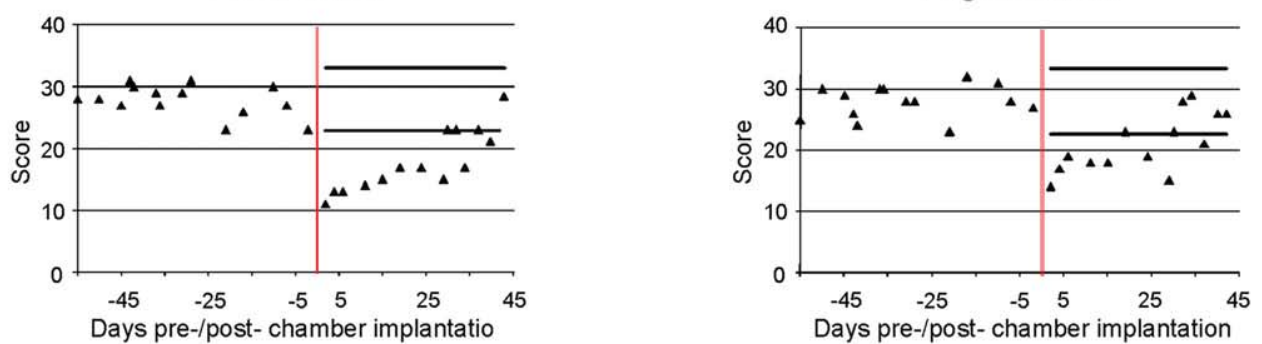

Control Mk-SL

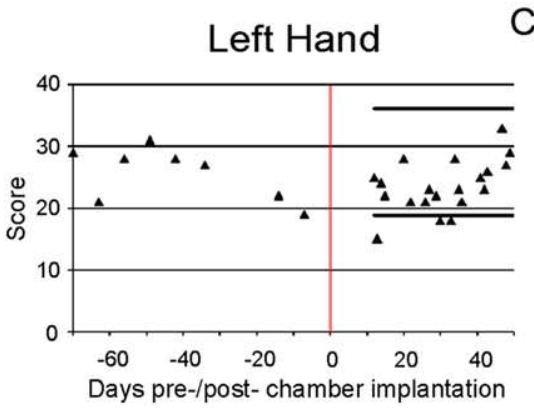

\section{Right Hand}

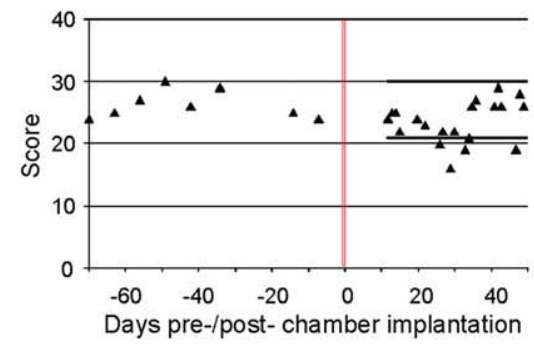

Right Hand

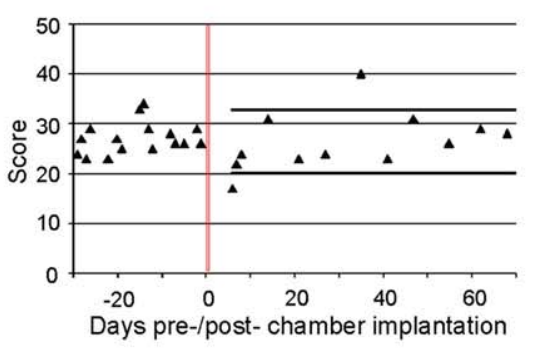

Control Mk-VA

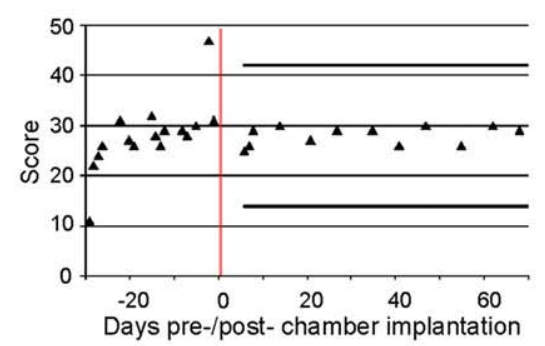

B

dIPFC lesioned Mk-AV

B Left (contralesional) Hand

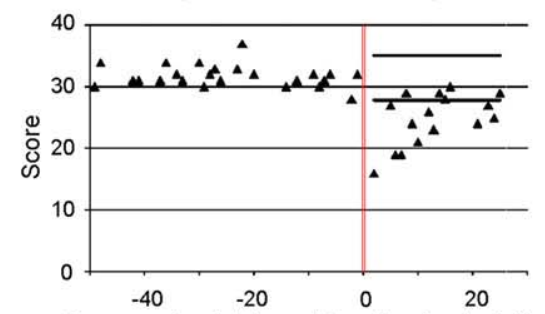

Days pre-/post- biopsy/chamber implantation
Right (ipsilesional) Hand

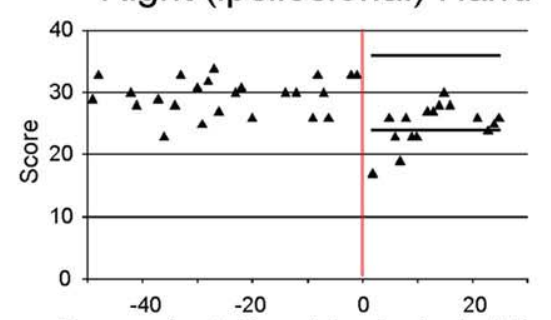

Days pre-/post- biopsy/chamber implantation

dIPFC lesioned Mk-JO

\section{Left (contralesional) Hand}

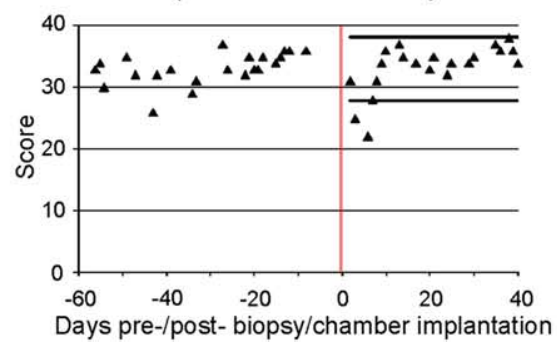

Right (ipsilesional) Hand

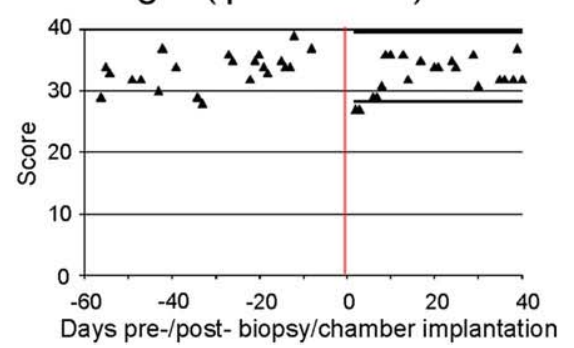

Fig. 3 - Number of pellets retrieved in $30 \mathrm{sec}$ using either hand (score; $y$ axis) from the $\mathbf{5 0}$ slots (cumulating the vertical and horizontal slots) in the modified Brinkman board task, by the control monkeys (panel A) and by the monkeys subjected to dlPFC lesion (panel B), along daily sessions pre- and post-surgery ( $x$ axis). The control monkeys $(n=3)$ were subjected to 
the three other monkeys (Mk-JA, MK-VA and MK-SL) were not subjected to such prefrontal cortical biopsy, but were implanted with a chronic recording chamber on the left hemisphere, they were considered as control subjects for the purpose of this PFC lesion study. Comparisons were thus performed between the two monkeys subjected to the PFC biopsy and the recording chamber implantation (Mk-JO and Mk-AV) versus the three monkeys implanted with the recording chamber only (Mk-JA, Mk-VA and Mk-SL).

After each surgery, the monkey was under observation until coming out of the anaesthesia, about 30-60 min after interruption of the propofol perfusion, and started to eat and drink. The monkey was placed alone in a separate cage for a couple of days to allow better conditions for recovery, and received Carprofen (pills of Rymadil mixed with food) daily and Albipen ${ }^{\circledR}$ (subcutaneously) every 2 days during 1 week.

\subsection{Histology of the prefrontal area after biopsy}

Once the entire experiment was completed (effect of subsequent motor cortex lesion and recovery, see Bashir et al., 2012; Kaeser et al., 2010, 2011), the monkeys were sacrificed. They were first anesthetized with an intramuscular ketamine injection, and then received a lethal dose of sodium pentobarbital $(90 \mathrm{mg} / \mathrm{kg})$. Following transcardiac perfusion with .9\% saline $(400 \mathrm{ml})$, the perfusion was continued with fixative (3 L of $4 \%$ phosphate buffered paraformaldehyde in $.1 \mathrm{M}$ phosphate buffer, $\mathrm{pH}=7.6$ ) and solutions ( $2 \mathrm{~L}$ each) of the same fixative containing increasing concentrations of sucrose (10, 20 and $30 \%$ ). The brain was removed, dissected and stored in a sucrose solution (30\%) for 1.5-2 weeks.

Frozen sections were then cut in the frontal plane at a thickness of $50 \mu \mathrm{m}$. Five to eight series of sections were collected with a cryotome (HM560, MICROM, Volketswil, Switzerland) and were stored at $-20{ }^{\circ} \mathrm{C}$, until used, in the cryoprotective $50 \mathrm{mM}$ phosphate buffer $\mathrm{pH} 7.4$ solution that contains 25\% glycerol (G7893, Sigma-Aldrich, St. Louis, MO, USA), 30\% ethylene glycol (33068, Ridel-de-Haën, Seelze, Germany). Among these series, one was Nissl-stained.

As described previously (Kaeser et al., 2011), histological analyses and reconstructions of the dlPFC lesion in the frontal plane were performed on these Nissl-stained sections with Mercator $^{\circledR}$. Three-dimensional mapping and volume quantifications were then performed with Map3D ${ }^{\circledR}$.

\section{Results}

\subsection{Histology}

In both Mk-JO and Mk-AV, the cortical biopsy included parts of the areas 9 and 46 of the dlPFC (Fig. 2). The quantification of the biopsy size revealed a larger lesion in $\mathrm{Mk}-\mathrm{AV}\left(44 \mathrm{~mm}^{3}\right)$ than in Mk-JO $\left(20.3 \mathrm{~mm}^{3}\right)$. This was due to the mechanical removal of the tissue performed without precise definition of the volume to extract, but with the aim to remove little tissue, but enough for the cell cultures (Brunet et al., 2005; Kaeser et al., 2011).

\subsection{Motor performance}

Using the score parameter (number of pellets retrieved in $30 \mathrm{sec}$ ) in the modified Brinkman board task, the post-surgery performance was compared to the pre-surgery one based on the scores for all wells (cumulating vertical and horizontal slots). A mean score and a SD values were computed for the pre-surgery phase. In the post-surgery phase, data points situated outside the mean plus or minus 2 SDs area were considered as significant deviations from pre-surgery manual dexterity performance (Fig. 3). In the control monkeys (chronic chamber implantation only, see Fig. 3A), a significant but reversible decrease of performance was observed after the implantation of the chronic chamber on the left hemisphere in Mk-JA, for both the left and the right hands, lasting about 30 days. In the other two control monkeys (Mk-SL and Mk-VA), the decrease of score post-implantation of the chronic chamber was either non-significant or only very transient (limited to a few daily sessions). In the dlPFC lesioned monkeys (Fig. 3B), an effect of the surgery (chronic chamber implantation and dlPFC biopsy) on behavioural score was also observed, but to the same extent as in the control monkeys. The extent of the decrease of score in Mk-AV is comparable to that observed in the control Mk-JA, but recovered more rapidly after about 10 days. In the second monkey subjected to dlPFC lesion (Mk-JO), the decrease of score was modest and shortlasting, as in the control monkeys Mk-SL and Mk-VA.

When looking at the contact time in the Modified Brinkman Board task (data not shown), as for the score, a statistically significant but transient effect of the surgery, reflected by an increase of contact time independently of the wells orientation, was observed both in the control and the dlPFC lesioned monkeys. To note that this effect concerned mainly the right hand, suggesting that it may be mostly related to the chronic chamber implantation on the left hemisphere.

In the rotating Brinkman board task (data not shown), various effects in terms of increase and of decrease of contact time were partially observed in the control monkeys. In the dlPFC lesioned monkeys, little statistically significant effect was found, namely uniquely an increase of contact time in $\mathrm{Mk}-\mathrm{AV}$ for the right hand when the board rotated counterclockwise.

Therefore, as these transient impacts on the score and the contact time were present in both groups of monkeys, they could not be attributed to the dlPFC lesion specifically, but may rather reflect a general drop of motor performance following any type of heavy surgery implicating deep anaesthesia, post-surgery treatment (e.g., the analgesic Carprofen) and/or the implantation of a chronic recording chamber unilaterally.

chronic chamber implantation only whereas the same implantation was combined to a unilateral lesion of dlPFC in the dlPFC lesion monkeys $(n=2)$. The red vertical line indicates the surgery day. The post-surgery performance was compared to the pre-surgery one, given by its mean value plus or minus 2 SDs. The two black horizontal lines indicate the superior and inferior limits (mean pre-lesion value plus 2 SDs and mean pre-lesion value minus 2 SD, respectively). 

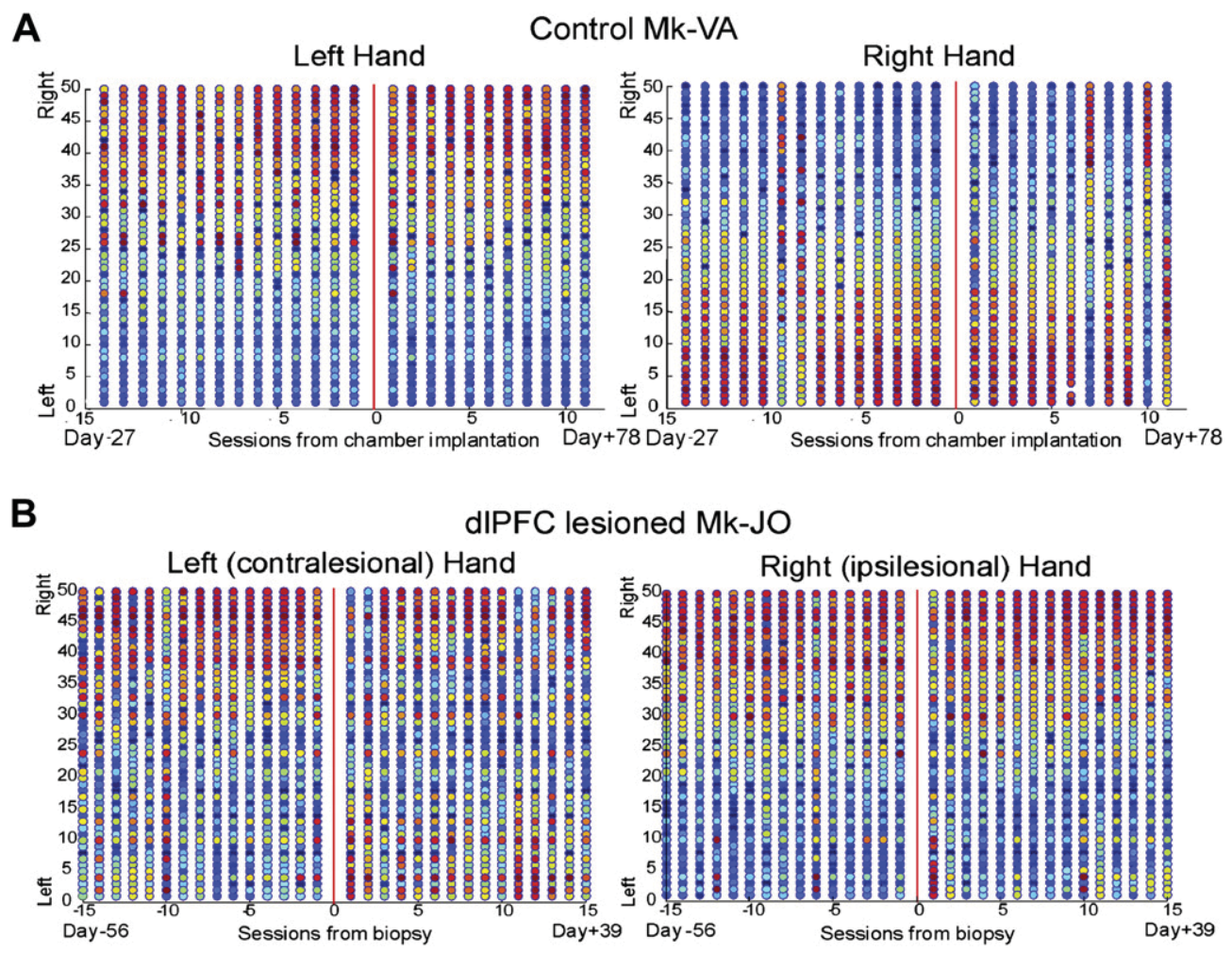

dIPFC lesioned Mk-AV

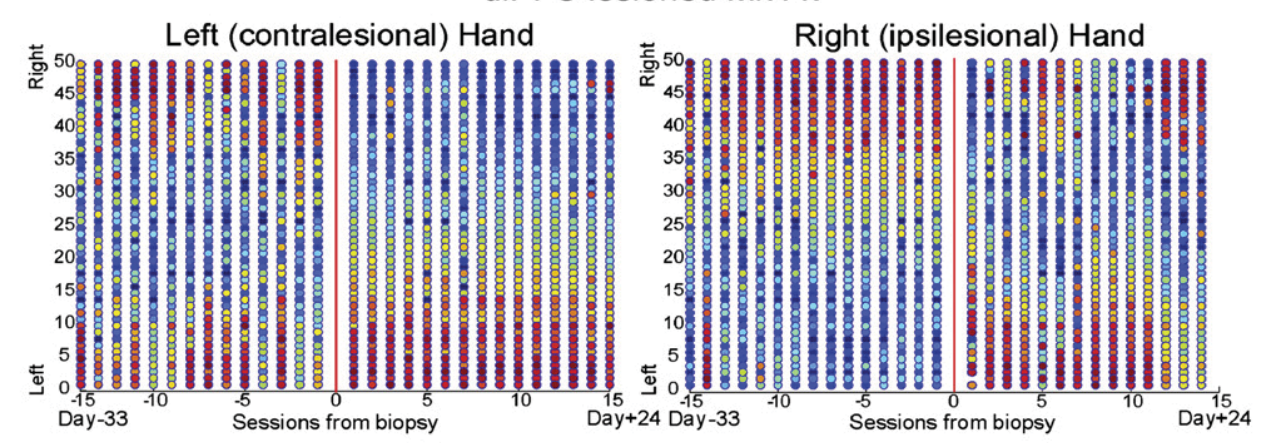

C

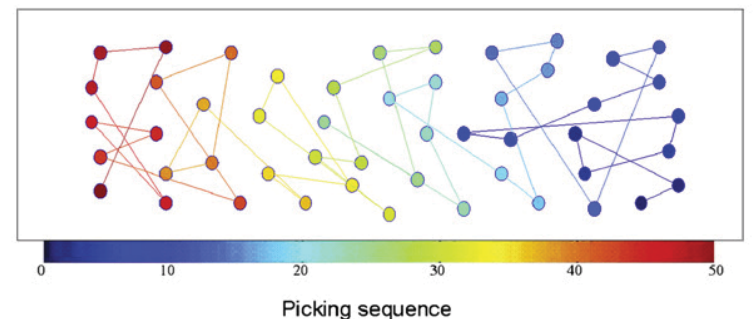

Fig. 4 - Picking sequence along the left-right axis in the modified Brinkman board task for both hands of one representative control monkey (A) and the two dlPFC lesioned monkeys (B). $X$ axis represents the consecutive behavioural sessions, one column thus corresponding to one daily session. $\mathrm{Y}$ axis represents the 50 wells of the board, ordered according to their position along the left-right axis, independently of their position along the vertical axis (see Fig. 1A). However, to avoid overlap of symbols, similar sequential numbers (e.g., the 2 wells numbered as 1.5 in Fig. 1A) were arbitrarily split as numbers 1 and 2 along the ordinate. Colours indicate the temporal picking sequence, ranging from 1 (blue; first pellet retrieved) to $\mathbf{5 0}$ (red; last pellet retrieved). Red vertical lines represent the surgery day. The board at the bottom of the Figure (C) represents the modified Brinkman board with its 50 wells, coloured and linked according to the prehension sequence in a given daily session. In the present example, the monkey began to pick the pellets from the right side of the board (blue symbols), and then progressively moved towards the left side, with red symbols at the leftmost extremity of the board (Mk-AV, left hand post-lesion). 

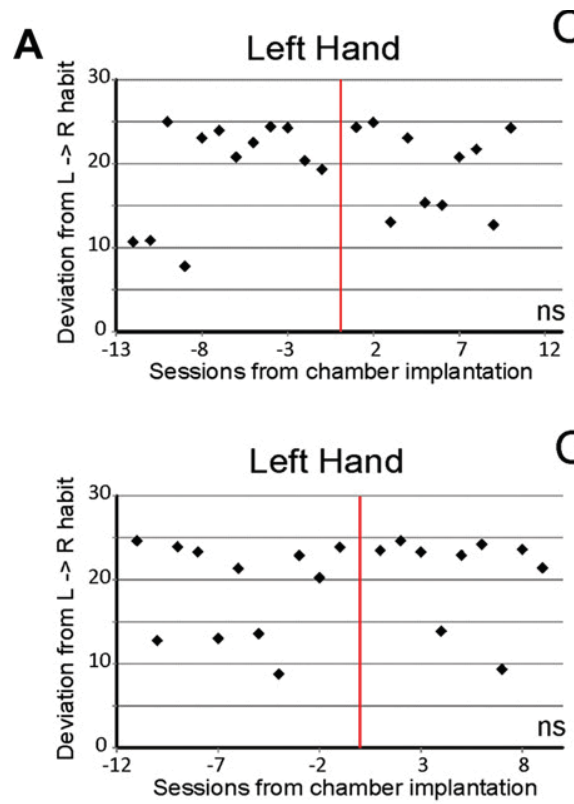

Control Mk-SL
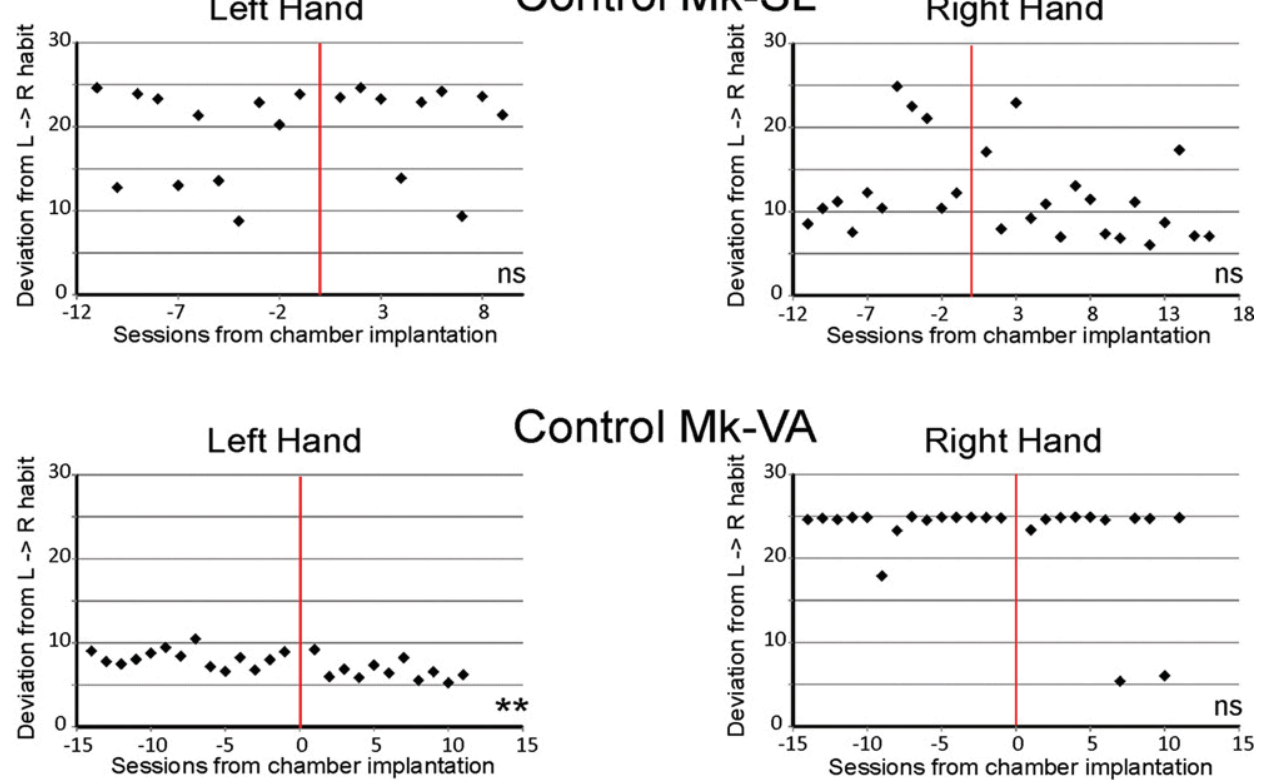

Control Mk-VA Right Hand
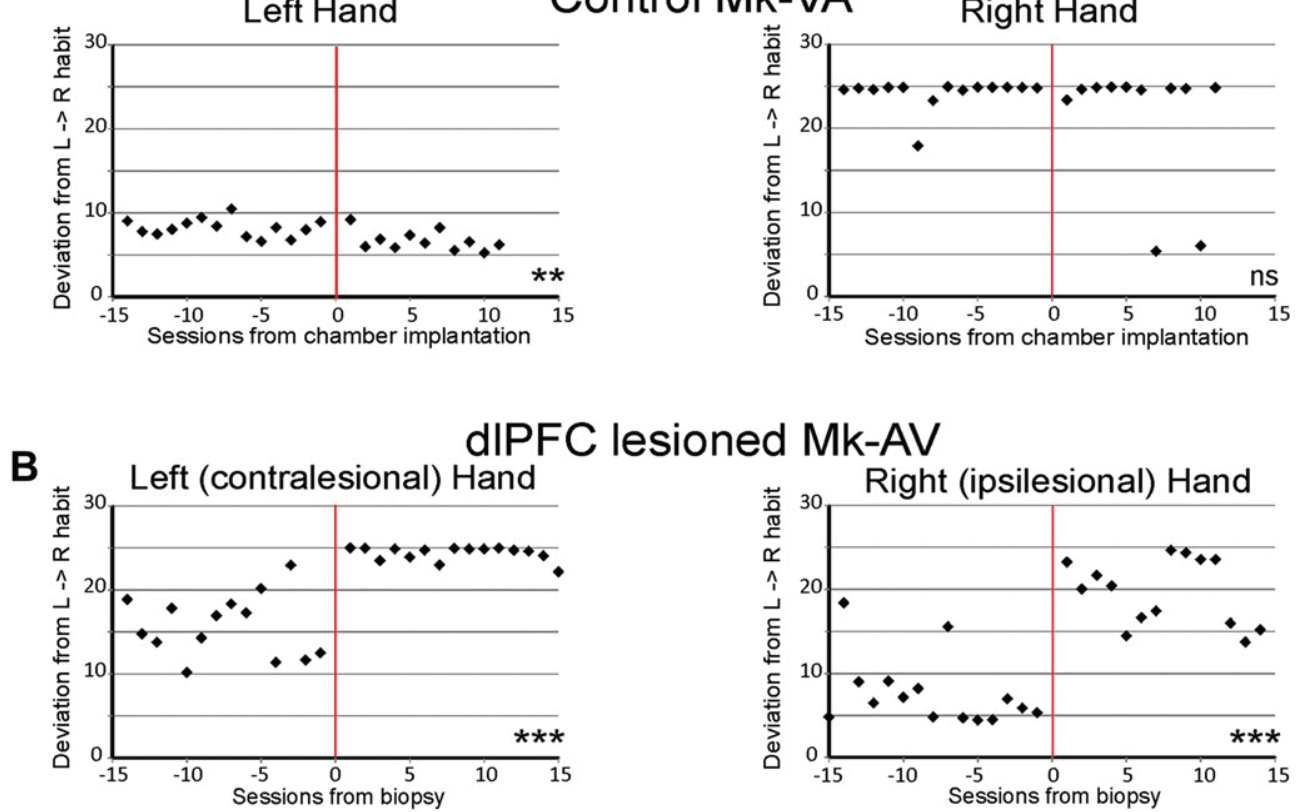

\section{lesioned Mk-AV}

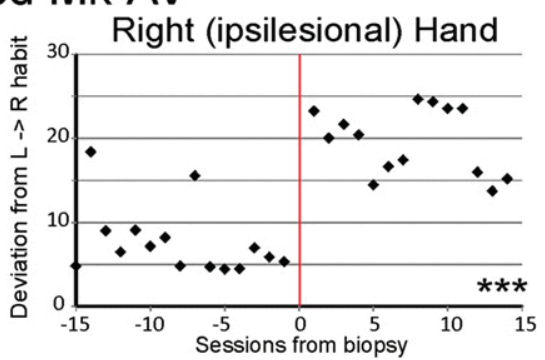

dIPFC lesioned Mk-JO
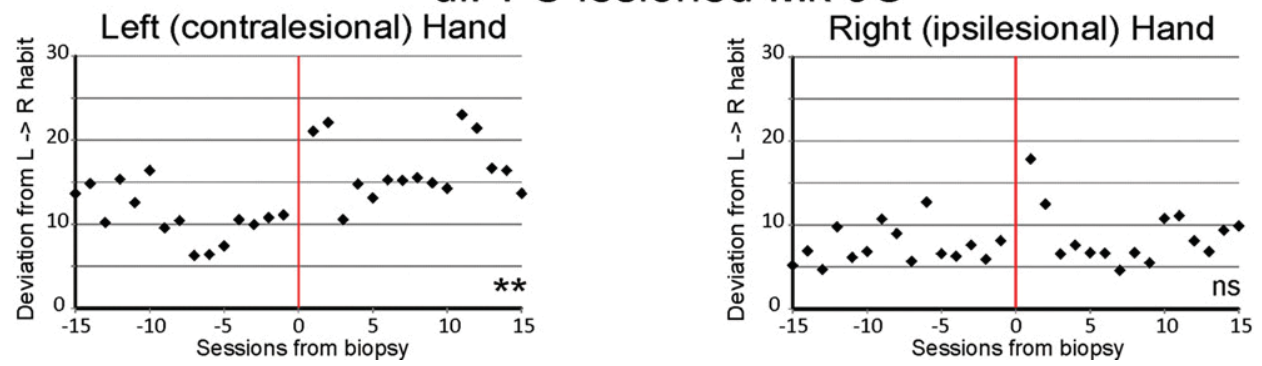

Fig. 5 - Quantitative assessment of motor sequence in the modified Brinkman board task for the three control monkeys (panel A) and the two monkeys subjected to unilateral dlPFC lesion (panel B). For each graph, as explained in the results section, an index of systematic motor sequence was computed and plotted as a function of behavioural session before and after the day ( 0 in the abscissa) of chamber implantation in the control monkeys and dlPFC lesion associated to chamber implantation in the 2 lesioned monkeys. In ordinate, the index of motor sequence indicates the extent of deviation from a systematic sequence starting from the left extremity of the board and terminating at its right extremity, corresponding to 


\subsection{Motor habit}

The parameters score and contact time considered above are related to the performance of the monkey in the manual dexterity task, as the score reflects the speed at which the monkey performs the task overall, whereas the contact time specifically reflects the manual manipulative component of the task. In contrast, the concept of motor habit is independent from the performance per se, as it rather reflects here the sequential order in which the wells are visited by the monkey. Although an optimal motor sequence may improve the performance, similar levels of performance can be reached by adopting different sequences, namely by starting on one side of the board and then systematically progressing towards the other extremity, or vice-versa.

\subsubsection{Modified Brinkman board task}

When looking at the picking sequence in the modified Brinkman board task, the qualitative data indicated that, before the surgery (biopsy and/or chronic chamber implantation), all monkeys performed the task following a more or less reproducible sequence. Indeed, the pellets were not retrieved randomly along the horizontal axis of the board (Fig. 4), although the sequence of prehension differed across the monkeys. For example, Mk-VA (control monkey), when performing the task with the left hand, retrieved the pellets situated on the left side of the board first (blue circles), moving gradually towards the central part (green and yellow circles) and finally towards the right part of the board (red circles), whereas this monkey performed the task in a "mirror" sequence with the right hand, namely by retrieving the pellets from the right side (blue circles) to the left side (red circles) of the board (Fig. 4A). Still before surgery (including dlPFC lesion), Mk-JO also exhibited a general trend with the left hand to scan the board from left to right (Fig. 4B), though a bit less systematic than Mk-VA. The same motor sequence (left to right scan) was adopted by Mk-JO for the right hand (Fig. 4B), in contrast to the right hand of Mk-VA (right to left scan). In a different way (Fig. 4B), before the lesion of dlPFC, Mk-AV grasped the pellets with the left hand from the centre (blue circles), moving towards sometimes the left, sometimes the right part of the board (green and yellow circles for the pellets grasped at the middle of the task, and red circles for the pellets retrieved towards the end of the task). This monkey, when performing the task with the right hand, retrieved first the pellets from the left side of the board first (blue circles), then those placed in the centre of the board (green and yellow circles), to finally end with the pellets located on the right side (red circles; Fig. 4B). The qualitative data in Fig. 4 show that the motor habit related to motor sequence varies from one animal to the next for the same manual dexterity task. Fig. 4 also shows qualitatively that the chronic chamber implantation did not modify the motor sequence in the control monkey MkVA, whereas the simultaneous chronic chamber implantation and dlPFC lesion changed the motor sequence in Mk-JO and Mk-AV.

The data as presented in Fig. 4 are limited to a qualitative assessment of motor habits adopted by the monkey regarding the sequence of prehension. To quantify motor sequencing, the data were processed as follows. In the modified Brinkman board (Fig. 1A), for each well, its spatial position number was subtracted from the temporal position in the sequence (1-50) without considering the sign of this difference. Finally, these differences calculated for the 50 wells were cumulated, providing an index of systematic motor sequence. For instance, for a monkey scanning systematically the modified Brinkman board from left to right along the horizontal axis, the cumulated difference is a small number as the temporal position of the wells in the sequence are close to the spatial number of the wells. On the other hand, going from right to left yields a large cumulated difference. This quantification allows also better assessing whether the monkey repeats the same sequence from one session to the next, as reflected in such a case by a small variability of the cumulated difference from one daily session to the next. The quantitative data for the modified Brinkman board are presented for all monkeys in Fig. 5. In the control monkeys, Mk-VA is a good example of a systematic motor sequence. With the left hand, Mk-VA scanned the board systematically from left to right (see also Fig. 4A), yielding small cumulated differences (representing a weak deviation from a left to right sequence of prehension). After chronic chamber implantation, the same sequence was maintained, even enforced as the cumulated difference was significantly smaller than pre-lesion. For the right hand, a large cumulated difference was found (Fig. 5A), as Mk-VA scanned the board systematically from right to left, with the exception of a couple of daily sessions in which the sequence was different both pre- and post-implantation of the chamber (see also Fig. 4A). There was no difference of cumulated differences between the sessions pre- and post-implantation of the chronic chamber. In the other two control monkeys (Mk-JA and Mk-SL), one animal (Mk-JA) showed a highly systematic motor sequence with the right hand only (scan from left to right). For the left hand, as well as either hand of Mk-SL, the motor sequence was less organized and systematic, without statistically significant difference between preand post-implantation of the chronic chamber (Fig. 5A).

In the two monkeys subjected to dlPFC lesion (and chronic chamber implantation), the quantitative data (Fig. 5B) show an effect on the motor sequence. In Mk-AV (with the largest lesion), the left (contralesional) hand exhibits a strong change of sequence when comparing the sessions before the lesion (sequence starting in the middle of the board) and the sessions after the lesion (sequence going systematically from right to

\footnotetext{
a low value for this precise sequence. The mirror sequence (right to left systematic scan) yields high values. A small variability from one daily behavioural session to the next indicates a reproducible motor sequence reflecting motor habit. See text for detailed description of the results. The index of motor sequence (ordinate) was compared pre- versus postsurgery in control monkeys and in dlPFC lesion monkeys, based on the non-parametric Mann and Whitney test. The result of the statistical comparison is indicated for each graph in the bottom right: $\mathrm{ns}=$ statistically non-significant difference $(p>.05) ;{ }^{*}$ is for $p \leq .05$; $^{* *}$ is for $p \leq .01$.
} 
A
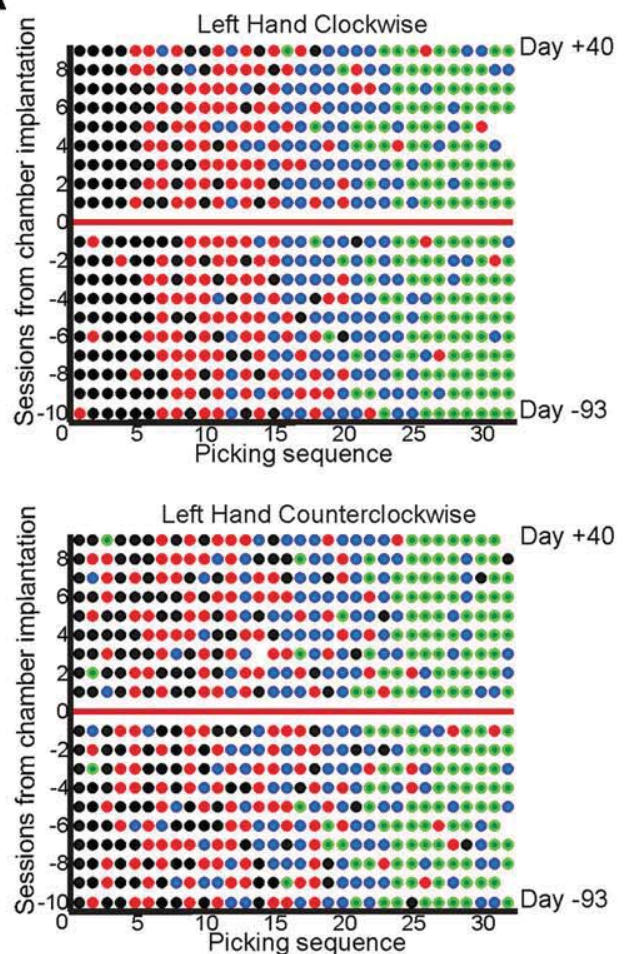

Right Hand Clockwise

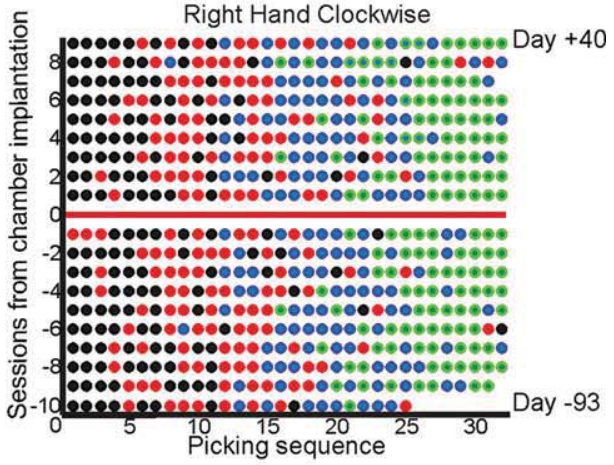

Right Hand Counterclockwise

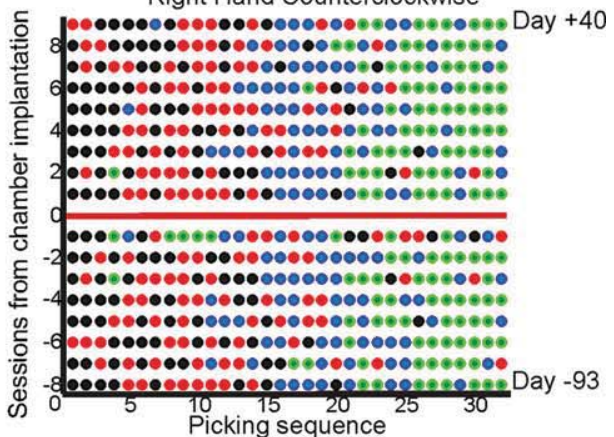

dIPFC lesioned Mk-AV

B
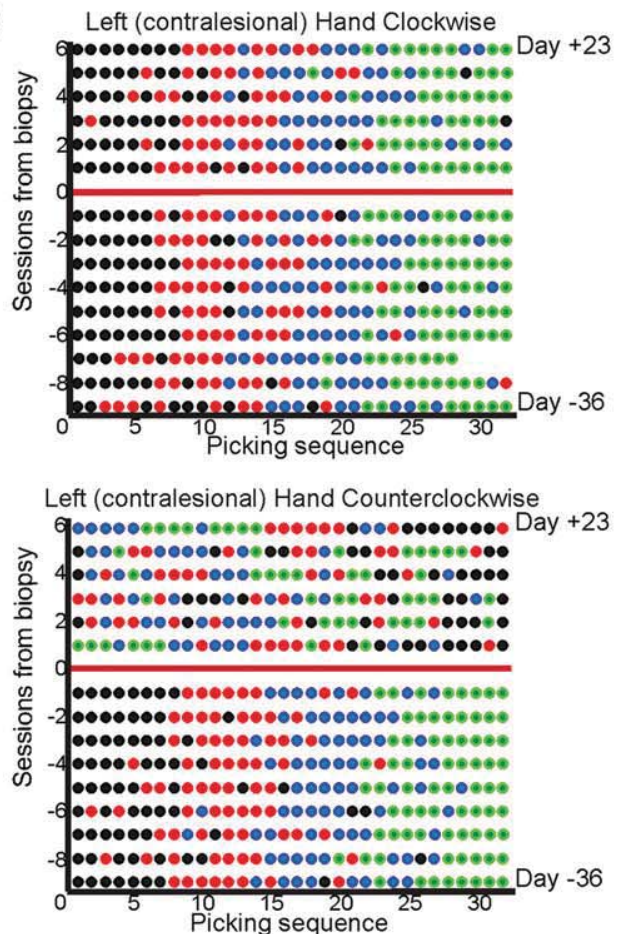

Right (ipsilesional) Hand Clockwise

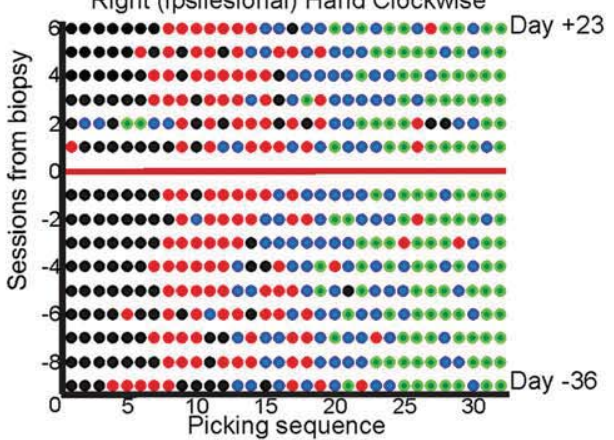

Right (ipsilesional) Hand Counterclockwise

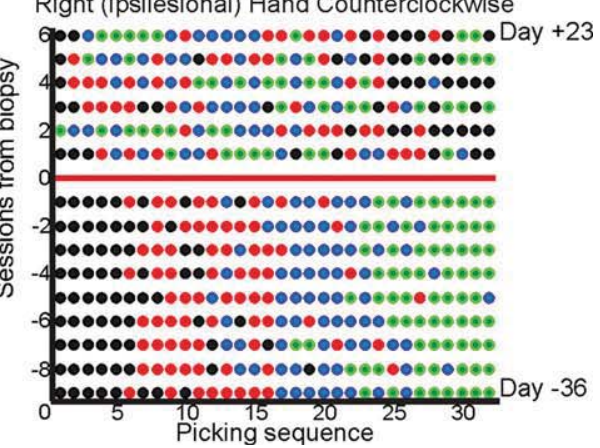

Fig. 6 - Picking sequence from the four rings of the rotating Brinkman board task for each hand of one representative control monkey and one representative PFC lesioned monkey. $X$ axis represents the picking sequence, each retrieval being coloured according to the corresponding ring (black for the first, red for the second, blue for the third and green for the fourth; see Fig. 1B). $Y$ axis indicates the consecutive behavioural sessions, one line thus corresponding to one daily session. Red horizontal lines represent the day of surgery. 

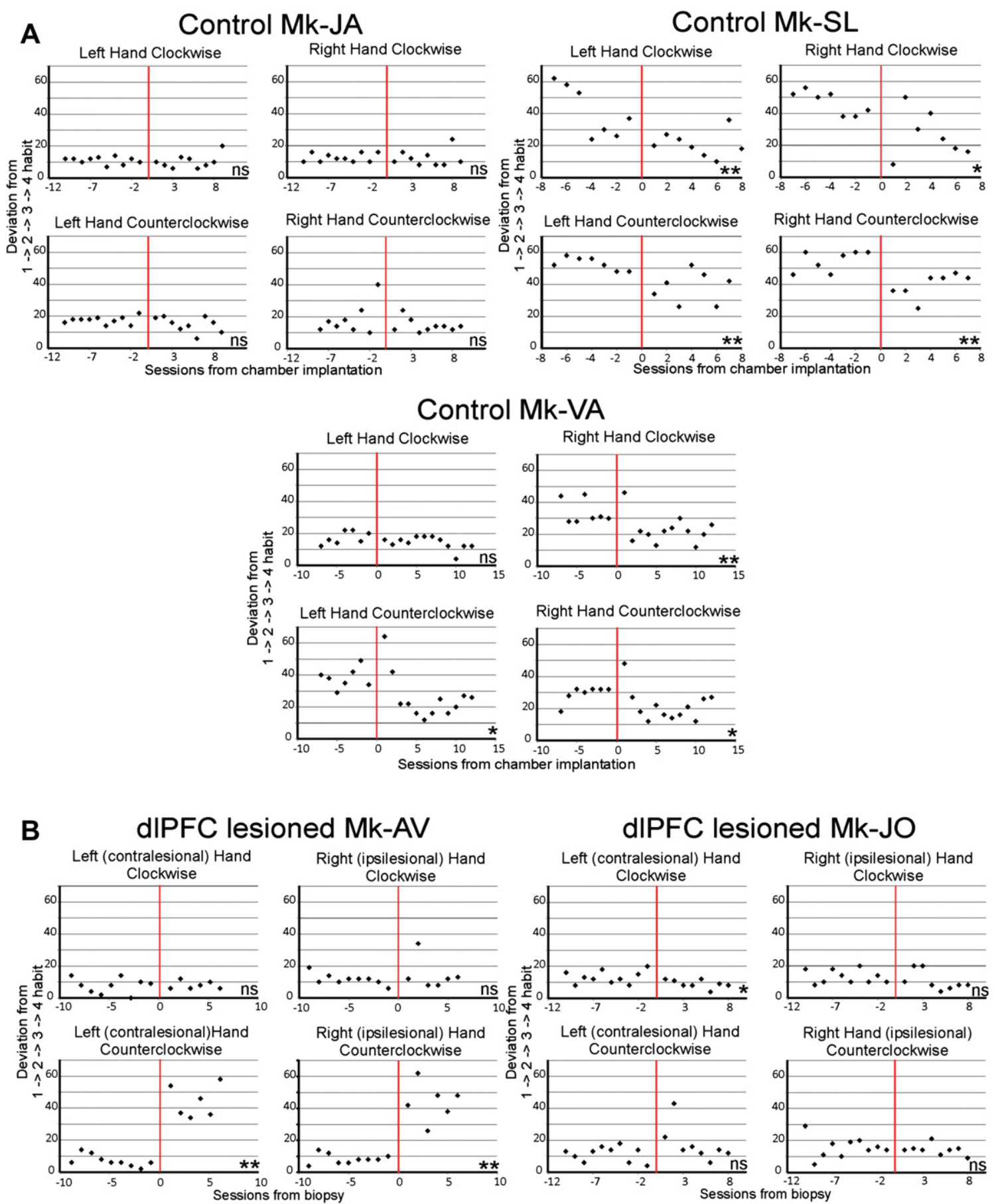

Fig. 7 - Quantitative assessment of motor sequence in the rotating Brinkman board task for the three control monkeys (panel A) and the two monkeys subjected to unilateral dlPFC lesion (panel B). For each graph, as explained in the results section, an index of systematic motor sequence was computed and plotted as a function of behavioural session before and after the day ( 0 in the abscissa) of chamber implantation in the control monkeys and dlPFC lesion associated to chamber implantation in the 2 lesioned monkeys. In ordinate, the index of motor sequence indicates the extent of deviation from a systematic sequence starting to empty the slots in the external ring and terminating in the inner ring, corresponding to a low value for this precise sequence. The opposite sequence (inner ring to external ring) would yield high values. A small variability from one daily behavioural session to the next indicates a reproducible motor sequence reflecting motor habit. The index of motor sequence (ordinate) was compared pre- versus post-surgery in control monkeys and in dlPFC lesion monkeys, based on the non-parametric Mann and Whitney test. The result of the statistical comparison is indicated for each graph in the bottom right: $n s=$ statistically non-significant difference $(p>.05) ;{ }^{*}$ is for $p \leq .05 ;{ }^{* *}$ is for $p \leq .01$. 
left). The difference is statistically highly significant (Man$\mathrm{n}$-Whitney test, $p<.001)$. Still in Mk-AV, a strong effect of the dlPFC lesion was also found for the right (ipsilesional) hand (Mann-Whitney test, $p<.001$ ), consisting in a switch from generally left to right sequence pre-lesion to the mirror general sequence (right to left) post-lesion, although there was some variability from one daily session to the next (Fig. 5B). In Mk-JO (subjected to a smaller dlPFC lesion), a significant change of motor sequence related to the dlPFC lesion was found for the left (contralesional) hand only (Mann-Whitney test, $p<.01$ ). Indeed, before lesion, Mk-JO progressively reinforced a left to right motor habit with the left hand, which turned into a more erratic motor sequence post-lesion (Figs. $4 \mathrm{~B}$ and $5 \mathrm{~B})$. In contrast, for the right (ipsilesional) hand, Mk-JO did not modify the motor sequence after lesion of dlPFC.

Overall, a clear change of prehension sequence in terms of picking pellets along the left-right axis occurred in the modified Brinkman board task in the dlPFC lesioned monkeys, except in Mk-JO for the right (ipsilesional) hand, whereas no such change was observed in the control monkeys in relation to the chronic chamber implantation. Therefore, this change of prehension strategy is most likely a consequence specific to the dlPFC lesion. Note that also in Mk-AV, with the largest dlPFC lesion, the monkey partially returned to the initial motor sequence with the right (ipsilesional) hand after about 11 daily sessions (Figs. 4B and 5B).

\subsubsection{Rotating Brinkman board task}

When looking at the prehension sequence from the four rings of the rotating Brinkman board task (Fig. 1B), before the surgery (Fig. 6), a general trend appeared, retrieving the pellets from the most external (either 1 - black circles - or 1 and $2-$ red circles - mixed) to the most internal ( 4 - green circles) rings. In other words, the monkey first retrieved the pellets that were the closest, except Mk-SL (Fig. 7A) showing a quite randomly distributed prehension sequence, grasping some pellets from the inner ring 4 (green circles) at the beginning of the session. Furthermore, in Mk-VA (Fig. 7A), the rings were more intermixed in the sequence of grasping, even with a trend to retrieve the pellets from the internal to the external rings when the board rotated counterclockwise, as well as clockwise for the right hand (Fig. 7).

As described above for the modified Brinkman board (Fig. 5), the qualitative data presented in Fig. 6 for the rotating Brinkman board in two monkeys have been processed in order to provide a quantitative assessment for all monkeys (Fig. 7). The rings of the rotating Brinkman board were numbered from external to internal and a numerical difference was computed for each well between the ring number and the position of the corresponding slot in the temporal sequence. Neglecting the sign, the differences were cumulated for the 32 slots of the rotating Brinkman board. A small cumulated difference reflects a systematic motor sequence going from external to internal slots, observed for most monkeys (Fig. 7). On the contrary, a scan from internal to external rings yields a large cumulated difference (e.g., Mk-SL in Fig. 7).

As far as the effect of chronic chamber implantation is concerned (control monkeys), Mk-JA exhibited no change of motor sequence pre- versus post-implantation (Fig. 7A; see also Fig. 6A). In the other two control monkeys, the results are rather mixed and correspond roughly to three events: (i) no change (Mann and Whitney test, non-significant difference = "ns", e.g., left hand for clockwise rotation in Mk-VA); (ii) in spite of a statistically significant difference (Mann and Whitney test: "*” is for $p \leq .05$ or "**" is for $p \leq .01$ ), there was a progressive drift of motor sequence, not influenced by the chamber implantation (e.g., Mk-SL for left hand both rotation directions and right hand clockwise); (iii) a change related to the chamber implantation in 4 instances (Mann and Whitney test: "** is for $p \leq .05$ or "***" is for $p \leq .01$; e.g., Mk-SL for the right hand counterclockwise and Mk-VA for counterclockwise both hands and clockwise right hand). Overall, in the group of control monkeys, considering each hand and the two directions of rotation, no change directly related to chamber implantation was observed in 8 instances out of a total of 12 (Fig. 7A), whereas there was a change only in 4 instances. In the two monkeys subjected to dlPFC lesion (Fig. 7B), Mk-JO with the smallest lesion exhibited no change in the motor sequence for the rotating Brinkman board task, except a modest reinforcement of the systematic external to internal rings sequence. In Mk-AV (large dlPFC lesion), the motor sequence was not affected by the lesion in the clockwise rotation whereas there was a dramatic change in the counterclockwise rotation for both hands, as the monkey adopted a more erratic motor sequence, quite variable from one daily session to the next (Fig. 7B; see also Fig. 6B).

\section{Discussion}

Based on the comparison between three control monkeys (chronic chamber implantation only) and two monkeys subjected to a lesion of the dlPFC (in addition to chronic chamber implantation), the present study provides evidence that dlPFC is involved in the representation of motor habits in a "freewill" sequential motor task of manipulating multiple objects with the hand. Indeed a lesion of dlPFC led to a change of the temporal order of grasping pellets from 32 or 50 spatially distinct slots in the modified Brinkman board task and in the rotating Brinkman board task, respectively, although the impact of the lesion was more striking in the former than in the latter test. In contrast, the lesion of dlPFC did not affect the motor performance per se, namely the fine manual dexterity and/or the speed of movements' execution. There is preliminary evidence that the extent of the dlPFC lesion effect on the motor sequence relates to the size of the lesion. Furthermore, for the largest unilateral lesion of dlPFC (Mk-AV), the change of motor habits affected both hand, whereas in the case of a smaller dlPFC lesion (Mk-JO) the change was observed for the contralesional hand only. The change of motor habit related to dlPFC lesion was not just transient but lasted for at least 2-3 weeks (the evolution on the longer long-term is unknown, as the monkeys involved in our protocol were then subjected to a lesion of the primary motor cortex; see Kaeser et al., 2010, 2011). To the best of our knowledge, the present study represents a first evidence for a role of dlPFC in motor habits, such as motor sequencing on a "free-will" basis.

The role played by dlPFC in the sequential order of the present "free-will" manual dexterity task (modified Brinkman board task) in monkeys is reminiscent of previous data 
reported by Brinkman (1984), based on a similar task in relation to a unilateral lesion of SMA. As in the present study, before lesion of SMA, the monkeys performed the task in a systematic manner, going from one slot to the most adjacent one or to one close to it (Brinkman, 1984). After unilateral lesion of SMA, a short-term effect was observed, i.e., the trajectories of the hand movements visiting the different slots became more erratic, jumping from one slot to another distant one (Brinkman, 1984). In the latter study, this observation was made at a unique time point (1 week post-lesion) whereas, in the present study, the effect of the dlPFC lesion has been assessed at multiple time points post-lesion during a few weeks. In any case, both the study of Brinkman (1984) and the present work suggest that SMA and dlPFC belong to the same brain network in which sequential motor habits are represented. The representation of such motor habits, not in a single cortical area, but rather in multiple cortical areas (dlPFC and SMA at least), without excluding subcortical representation (e.g., basal ganglia, cerebellum), forming a brain network is consistent with the idea that the lesion of dlPFC affected the corresponding network by switching the motor sequence but also suggests that rapid post-lesion adaptation in the network allowed to represent the "new" motor sequence emerging after the lesion of dlPFC. A role of subcortical areas in the representation of motor habits remains an open question, although it was recently shown that the globus pallidus does not contribute to motor sequencing but to motor execution per se (Desmurget and Turner, 2010).

Is there a relationship between motor performance (score, contact time) and motor sequencing, for instance a significant drop of score associated to a change of motor habit regarding sequencing? From the present data, it does not seem to be the case. First, the change of motor performance after the dlPFC lesion and chamber implantation was very modest (Fig. 3: MkJO and Mk-AV), making unlikely an effect on the motor habit. Second, the duration of the drop in motor performance (a few days) before recovery of the score is different from the time course of the change of motor sequence (at least 2-3 weeks).

Can the change in motor habit following lesion of dlPFC be interpreted as a consequence of a spatial neglect centred on the object? Such a deficit is commonly associated to a lesion of the posterior parietal cortex, but as dlPFC is part of the same network such interpretation may be considered. Looking at the data presented in Fig. 4B, it is however unlikely. In Mk-JO, a right dlPFC lesion would correspond to a spatial neglect of the left half of the modified Brinkman board. Although after the dlPFC lesion, Mk-JO initiated grasping more on the right side instead of more systematically on the left side as prelesion, a few daily sessions clearly demonstrate that the left side of the board was not neglected with the left hand and even more so with the right hand, with which the scan continued to be initiated on the left side of the board, with the exception of the first post-lesion session. In Mk-AV, the change of motor habit with the left hand after dlPFC lesion may suggest a spatial neglect (onset in the centre of the board switched to the right extremity). However, with the right hand, although the monkey switched from a left to right sequence pre-lesion to a mirror trajectory post-lesion (right to left), there are again some daily sessions in which a few slots located on the left side of the board were visited early in the sequence (Fig. 4B).
Moreover, the present results demonstrate transient motor deficits of manual dexterity following a surgery, performed under deep anaesthesia and followed by post-surgery medication (analgesic and antibiotics), aimed at implanting a chronic recording chamber above the motor cortex unilaterally. The difficulty here is to tentatively distinguish the possible effect of the general anaesthesia and subsequent medication from the effect of the presence of a chronic chamber above the primary motor cortex (M1). In control monkeys (subjected only to the anaesthesia/medication and unilateral chronic chamber implantation), the score was diminished during a few days post-surgery. As this effect was comparable for each hand, it rather argues for a non-specific impact, possibly resulting from the general anaesthesia/ medication. In contrast, the effect on the contact time was more prominent on the hand opposite to the hemisphere where the chronic chamber was implanted, suggesting in addition an effect of the craniotomy and exposing the dura mater above M1. In summary, following implantation of a chronic chamber, a mixed effect of general anaesthesia/ medication and surgical intervention above a restricted cortical zone both played a role in transiently decreasing the motor performance. As a consequence, it is important to evaluate the motor performance of the monkeys continuously and, after chronic chamber implantation, to wait for a return to a behavioural plateau as pre-implantation, before pursuing the experimental protocol (e.g., lesion and/or electrophysiological recordings).

The effects on the motor performance observed after the surgery were not specifically related to the dlPFC lesion. Indeed, these changes occurred both in the control monkeys and the dlPFC lesioned monkeys, for both manual dexterity tasks. In the modified Brinkman board task, a transient decrease of motor performance on the score parameter was found systematically in all monkeys and for both hands. For the contact time, such a transient decrease of performance appeared both in control and dlPFC lesioned monkeys, especially for their right hand, again suggesting that the chamber implantation on the left hemisphere played a role. For the contact time in the rotating Brinkman board task, some changes were observed in all the control monkeys, but not systematically, whereas a change occurred in one of the two PFC lesioned monkeys (Mk-AV), affecting the right hand when the board rotated counterclockwise.

In contrast, when considering the temporal sequence of the multiple grasping (sequential order to visit the consecutive wells), the changes observed were specifically related to the right dlPFC biopsy. Indeed, for the modified Brinkman board task, there was a clear change of the picking sequence along the left-right axis of the board for the two hands in the dlPFC lesioned monkeys uniquely. The effect was more pronounced in $\mathrm{Mk}-\mathrm{AV}$, the monkey subjected to the largest biopsy ( $44 \mathrm{~mm}^{3}$, versus $20.3 \mathrm{~mm}^{3}$ in Mk-JO). Along the same line, the most significant change that happened in the rotating Brinkman board task concerned Mk-AV for the picking sequence from the four rings, when the board rotated counterclockwise, and for both hands. Although the present data are limited to two monkeys with a lesion of the right dlPFC, the comparison performed with three control monkeys showed a clear distinction between the two groups, a conclusion 
supported by statistical analysis (Figs. 5 and 7). Furthermore, the effects of the dlPFC biopsy were more substantial in the monkey subjected to the largest dlPFC lesion (Mk-AV us MkJO), supporting the notion that the effect on motor habit is most likely due to the dlPFC lesion.

Overall, apparently in line with previous studies having demonstrated an implication of the dlPFC in the visuo-spatial working memory (e.g., Courtney et al., 1998; Funahashi et al., 1993; Smith and Jonides, 1999; Qi et al., 2010; Wilson et al., 1993), the present results show changes resulting from right dlPFC lesion, mainly on parameters reflecting a processing of either static or rotation-moving visuo-spatial information, in relation to prehension movements and the planning of the optimal picking sequence to perform manual prehension tasks, of which execution depends on dlPFC (e.g., Amiez and Petrides, 2007; Barraclough et al., 2004; Fletcher et al., 1997; Goel and Dolan, 2000; Goldberg et al., 1994; Heekeren et al., 2006; Seo et al., 2007). Furthermore, the present results are consistent with the model of Petrides suggesting that dlPFC is involved in high order executive control functions, as well as with a model proposing a hemispheric laterality effect, with a greater right PFC activation during spatial tasks (Baker et al., 1996; McCarthy et al., 1996; Reuter-Lorenz et al., 2000). In the present study, the dlPFC biopsy was indeed performed in the right hemisphere. However, in absence of left dlPFC lesion in macaques involved in the present investigation, the issue of hemispheric lateralization in the macaque monkeys remains speculative.

The clear effect of the dlPFC lesion on the strategy of prehension of the monkeys in the two manual dexterity tasks is paradoxical, as one would have expected that with the overtraining for the two motor tasks, the planning of the temporal sequence of the wells to be visited, indeed under control of the dlPFC during training, would have been delegated to hierarchically lower brain areas (premotor cortex, M1, basal ganglia, etc.) when the motor tasks are over-trained. In other words, dlPFC is not only contributing to the development of habits during motor learning but, surprisingly, also to the persistence of such motor habits, at least as far as motor sequencing is concerned. An originality of the present study is to have demonstrated a role for the dlPFC in the control of a "free-will", over-trained sequential motor task, which is neither conditional nor dependent on working memory. Nevertheless, the data illustrated in Figs. 4-7 also show that such a role of dlPFC in motor habit can be taken over after the dlPFC lesion by other brain regions, as discussed above.

For the continuation of the experiments in the context of transplantation of autologous adult cortical cells, biopsies sampled from the prefrontal cortex have to be done to produce autologous progenitor neural cell ecosystems (Bloch et al., 2011; Brunet et al., 2002, 2003, 2005) both in a model of motor cortex lesion (Kaeser et al., 2010, 2011) and in a model of neurodegenerative disease (Brunet et al., 2009). The present data points out to the necessity to restrict the volume of the biopsy as much as possible (less than $10 \mathrm{~mm}^{3}$ ), to avoid an effect of the biopsy per se on the behavioural readout. Along this line, for future clinical trials, a compromise regarding the size of the biopsy needs to be found, between a sufficiently large biopsy to collect enough progenitor cells and a biopsy sufficiently small to limit undesired deficits, especially possible effects that were not assessed here, such as psychic state changes. The impacts of the dlPFC biopsy, observed here on the motor habit but not on the motor performance itself of monkeys, remains however limited when the volume of the biopsy is small (as in Mk-JO). These observations reinforce the conclusion from previous work that the autologous transplantation of adult progenitor cortical cells is a safe procedure, in addition to the consideration of improving recovery from cortical lesion in macaque monkeys (Kaeser et al., 2011).

\section{Acknowledgements}

Mélanie Kaeser, Thierry Wannier, Jean-François Brunet, Jocelyne Bloch, and Eric M. Rouiller designed the study, conducted the experiments, acquired the data, analysed and interpreted the data, and drafted the manuscript. Alexander F. Wyss conducted the experiments and acquired the data. All authors critically revised the text and significantly contributed to the final version of the manuscript.

Thanks are due to Dr. A. Belhaj-Saïf for his support and advices in the course of the experiments. The authors wish to thank the technical assistance of Laurence Grollimund, Catherine Pythoud, Véronique Moret, Christine Roulin, Françoise Tinguely and Christiane Marti (histology and behavioural evaluations), Josef Corpataux, Laurent Bossy, Bernard Bapst and Bernard Morandi (animal house keeping), André Gaillard (mechanics), Bernard Aebischer (electronics), Laurent Monney (informatics).

Grant Sponsors: Swiss National Science Foundation, grants No 31-61857.00, 310000-110005 and 31003A-132465 (EMR), No 3100A0-103924 (JB), the Novartis Foundation; the National Centre of Competence in Research (NCCR) on "Neural plasticity and repair" and the Christopher Reeves Foundation (Springfield, NJ).

The authors have no conflict of interest in relation to the present study.

\section{R E F E R E N C E S}

Amiez C and Petrides M. Selective involvement of the middorsolateral prefrontal cortex in the coding of the serial order of visual stimuli in working memory. Proceedings of the National Academy of Science USA, 104(34): 13786-13791, 2007.

Baker SC, Rogers RD, Owen AM, Frith CD, Dolan RJ, Frackowiak RS, and Robbins TW. Neural systems engaged by planning: A PET study of the Tower of London task. Neuropsychologia, 34(6): 515-526, 1996.

Barone P and Joseph JP. Prefrontal cortex and spacial sequencing in macaque monkey. Experimental Brain Research, 78(3): 447-464, 1989.

Barraclough DJ, Conroy ML, and Lee D. Prefrontal cortex and decision making in a mixed-strategy game. Nature Neuroscience, 7(4): 404-410, 2004.

Bashir S, Kaeser M, Wyss A, Hamadjida A, Liu Y, Bloch J, et al. Short-term effects of unilateral lesion of the primary motor cortex (M1) on ipsilesional hand dexterity in adult macaque monkeys. Brain Structure and Function, 217(1): 63-79, 2012.

Bates JF and Goldman-Rakic PS. Prefrontal connections of medial motor areas in the rhesus monkey. Journal of Comparative Neurology, 336(2): 211-228, 1993. 
Bengtsson SL, Ehrsson HH, Forssberg H, and Ullen F. Dissociating brain regions controlling the temporal and ordinal structure of learned movement sequences. European Journal of Neuroscience, 19(9): 2591-2602, 2004.

Berdyyeva TK and Olson CR. Rank signals in four areas of macaque frontal cortex during selection of actions and objects in serial order. Journal of Neurophysiology, 104(1): 141-159, 2010.

Bloch J, Kaeser M, Sadeghi Y, Rouiller EM, Redmond Jr DE, and Brunet JF. Doublecortin-positive cells in the adult primate cerebral cortex and possible role in brain plasticity and development. Journal of Comparative Neurology, 519(4): 775-789, 2011.

Boecker H, Dagher A, Ceballos-Baumann AO, Passingham RE, Samuel M, Friston KJ, et al. Role of the human rostral supplementary motor area and the basal ganglia in motor sequence control: Investigations with $\mathrm{H}_{2}{ }^{15} \mathrm{O}$ PET. Journal of Neurophysiology, 79(2): 1070-1080, 1998.

Brinkman C. Supplementary motor area of the monkey's cerebral cortex: short- and long-term deficits after unilateral ablation and the effects of subsequent callosal section. Journal of Neuroscience, 4(4): 918-929, 1984.

Brinkman J and Kuypers HGJM. Cerebral control of contralateral and ipsilateral arm, hand and finger movements in the splitbrain rhesus monkey. Brain, 96(4): 653-674, 1973.

Brunet JF, Pellerin L, Arsenijevic Y, Magistretti P, and Villemure JG. A novel method for in vitro production of human glial-like cells from neurosurgical resection tissue. Laboratory Investigation, 82(6): 809-812, 2002.

Brunet JF, Pellerin L, Magistretti P, and Villemure JG. Cryopreservation of human brain tissue allowing timely production of viable adult human brain cells for autologous transplantation. Cryobiology, 47(2): 179-183, 2003.

Brunet JF, Rouiller E, Wannier T, Villemure JG, and Bloch J. Primate adult brain cell autotransplantation, a new tool for brain repair? Experimental Neurology, 196(1): 195-198, 2005.

Brunet JF, Redmond Jr DE, and Bloch J. Primate adult brain cell autotransplantation, a pilot study in asymptomatic MPTP treated monkeys. Cell Transplantation, 18(7): 787-799, 2009.

Clower WT and Alexander GE. Movement sequence-related activity reflecting numerical order of components in supplementary and presupplementary motor areas. Journal of Neurophysiology, 80(3): 1562-1566, 1998.

Courtney SM, Petit L, Maisog JM, Ungerleider LG, and Haxby JV. An area specialized for spatial working memory in human frontal cortex. Science, 279(5355): 1347-1351, 1998.

Deiber MP, Honda M, Ibanez V, Sadato N, and Hallett M. Mesial motor areas in self-initiated versus externally triggered movements examined with fMRI: Effect of movement type and rate. Journal of Neurophysiology, 81(6): 3065-3077, 1999.

Desmurget $\mathrm{M}$ and Turner RS. Motor sequences and the basal ganglia: kinematics, not habits. Journal of Neuroscience, 30(22): 7685-7690, 2010.

Dum RP and Strick PL. The origin of corticospinal projections from the premotor areas in the frontal lobe. Journal of Neuroscience, 11(3): 667-689, 1991.

Dum RP and Strick PL. Frontal lobe inputs to the digit representations of the motor areas on the lateral surface of the hemisphere. Journal of Neuroscience, 25(6): 1375-1386, 2005.

Fang PC, Stepniewska I, and Kaas JH. The thalamic connections of motor, premotor, and prefrontal areas of cortex in a prosimian primate (Otolemur garnetti). Neuroscience, 143(4): 987-1020, 2006.

Fletcher PC, Frith CD, and Rugg MD. The functional neuroanatomy of episodic memory. Trends in Neurosciences, 20(5): 213-218, 1997.

Freund P, Schmidlin E, Wannier T, Bloch J, Mir A, Schwab ME, et al. Anti-Nogo-A antibody treatment promotes recovery of manual dexterity after unilateral cervical lesion in adult primates-re-examination and extension of behavioral data. European Journal of Neuroscience, 29(5): 983-996, 2009.

Funahashi S, Chafee MV, and Goldman-rakic PS. Prefrontal neuronal activity in rhesus monkeys performing a delayed anti-saccade task. Nature, 365(6448): 753-756, 1993.

Gerloff C, Corwell B, Chen R, Hallett M, and Cohen LG. Stimulation over the human supplementary motor area interferes with the organization of future elements in complex motor sequences. Brain, 120(9): 1587-1602, 1997.

Goel V and Dolan RJ. Anatomical segregation of component processes in an inductive inference task. Journal of Cognitive Neuroscience, 12(1): 110-119, 2000.

Goldberg E, Podell K, and Lovell M. Lateralization of frontal lobe functions and cognitive novelty. Journal of Neuropsychiatry and Clinical Neurosciences, 6(4): 371-378, 1994.

Hadj-Bouziane F, Meunier M, and Boussaoud D. Conditional visuo-motor learning in primates: A key role for the basal ganglia. Journal of Physiology (Paris), 97(4-6): 567-579, 2003.

Halsband $U$ and Lange RK. Motor learning in man: A review of functional and clinical studies. Journal of Physiology (Paris), 99(4-6): 414-424, 2006.

Heekeren HR, Marrett S, Ruff DA, Bandettini PA, and Ungerleider LG. Involvement of human left dorsolateral prefrontal cortex in perceptual decision making is independent of response modality. Proceedings of the National Academy of Science USA., 103(26): 10023-10028, 2006.

Isoda $\mathrm{M}$ and Tanji J. Contrasting neuronal activity in the supplementary and frontal eye fields during temporal organization of multiple saccades. Journal of Neurophysiology, 90(5): 3054-3065, 2003.

Isoda $\mathrm{M}$ and Tanji J. Participation of the primate presupplementary motor area in sequencing multiple saccades. Journal of Neurophysiology, 92(1): 653-659, 2004.

Kaeser M, Wyss AF, Bashir S, Hamadjida A, Liu Y, Bloch J, et al. Effects of unilateral motor cortex lesion on ipsilesional hand's reach and grasp performance in monkeys: Relationship with recovery in the contralesional hand. Journal of Neurophysiology, 103(3): 1630-1645, 2010.

Kaeser M, Brunet JF, Wyss A, Belhaj-Saif A, Liu Y, Hamadjida A, et al. Autologous adult cortical cell transplantation enhances functional recovery following unilateral lesion of motor cortex in primates: A pilot study. Neurosurgery, 68(5): 1405-1416, 2011.

Kennerley SW, Sakai K, and Rushworth MF. Organization of action sequences and the role of the pre-SMA. Journal of Neurophysiology, 91(2): 978-993, 2004.

Kurata K. Corticocortical inputs to the dorsal and ventral aspects of the premotor cortex of macaque monkeys. Neuroscience Research, 12(1): 263-280, 1991.

Leichnetz GR. Afferent and efferent connections of the dorsolateral precentral gyrus (area 4 , hand/arm region) in the macaque monkey, with comparison to area 8. Journal of Comparative Neurology, 254(4): 460-492, 1986.

Lepage M, Beaudoin G, Boulet C, O’Brien I, Marcantoni W, Bourgouin P, et al. Frontal cortex and the programming of repetitive tapping movements in man: Lesion effects and functional neuroimaging. Brain Research. Cognitive Brain Research, 8(1): 17-25, 1999.

Liu Y and Rouiller EM. Mechanisms of recovery of dexterity following unilateral lesion of the sensorimotor cortex in adult monkeys. Experimental Brain Research, 128(1-2): 149-159, 1999.

Lu M-T, Preston JB, and Strick PL. Interconnections between the prefrontal cortex and the premotor areas in the frontal lobe. Journal of Comparative Neurology, 341(3): 375-392, 1994.

Matelli M, Camarda R, Glickstein M, and Rizzolatti G. Afferent and efferent projections of the inferior area 6 in the macaque monkey. Journal of Comparative Neurology, 251(3): 281-298, 1986.

McCarthy G, Puce A, Constable RT, Krystal JH, Gore JC, and Goldman-Rakic P. Activation of human prefrontal cortex during 
spatial and nonspatial working memory tasks measured by functional MRI. Cerebral Cortex, 6(4): 600-611, 1996.

Mushiake H, Inase M, and Tanji J. Selective coding of motor sequence in the supplementary motor area of the monkey cerebral cortex. Experimental Brain Research, 82(1): 208-210, 1990

Mushiake $\mathrm{H}$, Inase $\mathrm{M}$, and Tanji J. Neuronal activity in the primate premotor, supplementary, and precentral motor cortex during visually guided and internally determined sequential movements. Journal of Neurophysiology, 66(3): 705-716, 1991.

Ninokura Y, Mushiake H, and Tanji J. Integration of temporal order and object information in the monkey lateral prefrontal cortex. Journal of Neurophysiology, 91(1): 555-560, 2004.

Passingham RE. Attention to action. Philosophical Transactions of the Royal Society of London: Biological Sciences, 351(1346): 1473-1479, 1996.

Qi XL, Katsuki F, Meyer T, Rawley JB, Zhou X, Douglas KL, et al. Comparison of neural activity related to working memory in primate dorsolateral prefrontal and posterior parietal cortex. Frontiers in Systems Neuroscience, 4: 12, 2010.

Reuter-Lorenz PA, Jonides J, Smith EE, Hartley A, Miller A, Marshuetz C, et al. Age differences in the frontal lateralization of verbal and spatial working memory revealed by PET. Journal of Cognitive Neuroscience, 12(1): 174-187, 2000.

Rizzolatti G and Luppino G. The cortical motor system. Neuron, 31(6): 889-901, 2001.

Sakagami M and Watanabe M. Integration of cognitive and motivational information in the primate lateral prefrontal cortex. Annals of the New-York Academy of Science, 1104: 89-107, 2007.

Schmidlin E, Kaeser M, Gindrat AD, Savidan J, Chatagny P, Badoud S, et al. Behavioral assessment of manual dexterity in non-human primates. Journal of Visualized Experiments, 57: 3258, 2011.

Schubotz RI and Von Cramon DY. Interval and ordinal properties of sequences are associated with distinct premotor areas. Cerebral Cortex, 11(3): 210-222, 2001.
Seo H, Barraclough DJ, and Lee D. Dynamic signals related to choices and outcomes in the dorsolateral prefrontal cortex. Cerebral Cortex, 17(Suppl. 1): i110-117, 2007.

Shima $\mathrm{K}$ and Tanji J. Both supplementary and presupplementary motor areas are crucial for the temporal organization of multiple movements. Journal of Neurophysiology, 80(6): 3247-3260, 1998

Shima K and Tanji J. Neuronal activity in the supplementary and presupplementary motor areas for temporal organization of multiple movements. Journal of Neurophysiology, 84(4): 2148-2160, 2000

Shima K, Isoda M, Mushiake H, and Tanji J. Categorization of behavioural sequences in the prefrontal cortex. Nature, 445(7125): 315-318, 2007.

Smith EE and Jonides J. Storage and executive processes in the frontal lobes. Science, 283(5408): 1657-1661, 1999.

Sohn JW and Lee D. Order-dependent modulation of directional signals in the supplementary and presupplementary motor areas. Journal of Neuroscience, 27(50): 13655-13666, 2007.

Tanaka S, Honda M, Hanakawa T, and Cohen LG. Differential contribution of the supplementary motor area to stabilization of a procedural motor skill acquired through different practice schedules. Cerebral Cortex, 20(9): 2114-2121, 2010.

Tanji J and Shima K. Role for supplementary motor area cells in planning several movements ahead. Nature, 371(6496): 413-416, 1994.

Van Mier Hi, Perlmutter JS, and Petersen SE. Functional changes in brain activity during acquisition and practice of movement sequences. Motor Control, 8(4): 500-520, 2004.

Verwey WB, Lammens R, and Van HJ. On the role of the SMA in the discrete sequence production task: A TMS study. Transcranial magnetic stimulation. Neuropsychologia, 40(8): 1268-1276, 2002.

Wilson FA, Scalaidhe SP, and Goldman-Rakic PS. Dissociation of object and spatial processing domains in primate. Science, 260(5116): 1955-1958, 1993. 